REVISTA DE LA ESCUELA DE CIENCIAS DE LA EDUCACIÓN, AÑO 16, NRO. 15, VOL. 2, JULIO A DICIEMRE DE 2020. PÁGINAS 1-9. ISSN 2362-3349 (EN LÍNEA). INFLUENCIA DEL LIDERAZGO PEDAGÓGICO EN LA PRÁCTICA DOCENTE. MARÍA EUGENIA UGALDE VILLALOBOS. ALBA LUZ CANALES GARCÍA.

\title{
INFLUENCIA DEL LIDERAZGO PEDAGÓGICO EN LA PRÁCTICA DOCENTE
}

\author{
María Eugenia Ugalde Villalobos* \\ Universidad Nacional, Escuela de Secretariado Profesional, Costa Rica \\ maria.ugalde.villalobos@una.ac.cr \\ Alba Luz Canales García** \\ Universidad Nacional, División de Educología, Costa Rica \\ alba.canales.garcia@una.ac.cr
}

Recibido: 6/09/2019 Aceptado: 2/12/2019

\section{Resumen}

El objetivo principal de este artículo consiste en identificar el liderazgo pedagógico y su influencia en la práctica docente desarrollada por los estudiantes de IV nivel de Educación Comercial, carrera compartida entre la Escuela de Secretariado Profesional y la División de Educología de la Universidad Nacional de Costa Rica.

La metodología empleada es de tipo cualitativo, tipo fenomenológico dado que describe la realidad vivida en torno a la práctica docente de los estudiantes, posteriormente, se contrastan los datos recolectados con la fundamentación teórica. Esta experiencia académica surge a raíz de la vivencia del curso y del desarrollo de la práctica en diferentes instituciones educativas.

Los resultados evidencian el significado de liderazgo para el estudiante practicante, para el profesor colaborador, al igual que los profesores de la carrera durante el proceso de formación. Además, se perciben las características de liderazgo y de motivación, así como la influencia del liderazgo pedagógico en el estudiantado. Dentro de los hallazgos más relevantes están los tres tipos de liderazgo, la motivación y comunicación asertiva entre otros.

Palabras clave: Liderazgo pedagógico - Pedagogía - Educación comercial y comunicación asertiva.

\section{Abstract}

The main objective of this article is to identify pedagogical leadership and its influence on the teaching practice developed by students from level four of Commercial Education, a major shared between the School of Professional Secretariat and the Division of Educology of National University of Costa Rica.

\footnotetext{
* Académica e investigadora en la Escuela de Secretariado Profesional de la Universidad Nacional, Costa Rica. Trabajó como profesora de Español en el Ministerio de Educación Pública y correctora de estilo en el periódico La Nación. Bachiller en Filosofía Española de la Universidad de Costa Rica. Magister en Educación con énfasis en Docencia Universitaria, Universidad Nacional.

** Académica e investigadora en la División de Educología del Centro de Investigación y Docencia en Educación CIDE, de la Universidad Nacional de Costa Rica, trabajó en el Ministerio de Educación Pública. Actualmente labora en la División de Educología en el área de la Educación Comercial y en proyectos: Fortalecimiento de la Mediación Pedagógica y en procesos de Autoevaluación de la Carrera de Educación Comercial. Licenciada en Educación Comercial, Magister en Educación con énfasis en Docencia Universitaria, Universidad Nacional.
} 
The methodology used is qualitative, a phenomenological type that describes the reality lived around the students' teaching practice. Subsequently, the data collected is contrasted with the theoretical basis. This academic experience arises from the participation in the course and the development of the practice in different educational institutions.

The results highlight the significance of leadership for the practicing student, for the collaborating teacher, as well as for the teachers of the major during the training process. In addition, there are some leadership and motivational characteristics perceived, as well as the influence of pedagogical leadership on the student. Among the most relevant findings are the three types of leadership, motivation and assertive communication among others.

Keywords: Pedagogical leadership - Pedagogy - Commercial education and assertive communication.

\section{Introducción}

El curso "Desafíos Didácticos en la Práctica Docente para la Educación Comercial" se imparte en el primer semestre de cada año en la División de Educología, carrera compartida con la Escuela de Secretariado Profesional de la Universidad Nacional. Dicha experiencia consiste en que el estudiantado a nivel de bachillerato realiza su práctica en una institución educativa a nivel de secundaria, es decir, en colegios a nivel público de la especialidad de Secretariado. Cada estudiante es supervisado por un profesor de la institución educativa (profesor colaborador), el cual tiene comunicación con el docente del curso Desafíos Didácticos de la Universidad, con el fin de conocer el proceso de la práctica de cada estudiante.

Para realizar este estudio, en 2017, la población estudiantil consultada contestó un cuestionario cuyo propósito fue conocer cómo influye el liderazgo tanto de los docentes colaboradores, como el del profesor del curso, las unidades académicas de la carrera, lo mismo que el de dicha población practicante.

El liderazgo, de alguna manera, influye en el estudiantado durante el desarrollo del proceso de enseñanza y aprendizaje. En otras palabras, liderazgo es la acción para involucrarse en el quehacer estudiantil, por lo tanto, el objetivo general de este artículo es analizar cómo se desarrolla el liderazgo pedagógico en el curso "Desafíos Didácticos en la Práctica Docente" con el fin de socializar sus implicaciones en el campo educativo. Los objetivos específicos son los siguientes: describir la percepción del estudiantado sobre el tema de liderazgo pedagógico en la práctica docente, clasificar los tipos de liderazgo que se desarrollan en el aula y contrastar la información que suministran los estudiantes con la teoría. Por lo anterior, se plantea la siguiente pregunta: ¿Cómo influye el liderazgo pedagógico en la práctica docente dirigida de la carrera de Educación Comercial de la Universidad Nacional?

\section{Marco teórico}

Para efectos de esta investigación, se procederá a definir los tipos de liderazgo con el fin de analizar cada uno de ellos en relación con el que se presenta en el curso "Desafíos Didácticos en la Práctica Docente".

Se considera importante iniciar con el significado de la palabra Educar, que como lo menciona Jové (2018) tiene dos significados "educere, que significa (conducir, guíar) y educare que significa (formar e instruir)" (Jové, 2018, p.24).

La carrera de Educación Comercial se puede definir como una carrera compartida en la cual los estudiantes reciben cursos propios del área, así como del componente pedagógico, los grados académicos que ofrece son diplomado, bachillerato y licenciatura.

Otro término que complementa este trabajo es la palabra líder, según Chinchilla, Jiménez y García (2018), el líder "es la persona en quien podemos confiar, porque es fiable y madura: sabe responder ante cualquier situación ordenando las situaciones propias y ajenas. El verdadero líder sabe dirigir a otros lucha constantemente por liderarse a sí mismo". Para Lecaros, (2017) el liderazgo pedagógico:

(...) es la cualidad esencial para diseñar, organizar y mejorar el desempeño docente a través del adelanto conjunto de competencias pertinentes y oportunas para el trabajo pedagógico basado en conocimientos y experiencias orientadas al dominio de habilidades pedagógicas significativas en los directivos de los centros educativos. Es importante recalcar, que las buenas prácticas pedagógicas en el aula conducen a un mayor aprendizaje de los estudiantes; por consiguiente, la potenciación del liderazgo pedagógico entre docentes aumentará los niveles de estimulación, aliento y crecimiento de toda la organización educativa (Lecaros, 2017, p.23) 
De acuerdo con el liderazgo pedagógico, según Rodríguez (2011), a nivel de escuelas, se desempeña un rol altamente significativo en el desarrollo de cambios en las prácticas docentes, en la calidad de ellas y en el impacto que presentan en el aprendizaje del alumnado. Asimismo, en el liderazgo de las escuelas eficaces, las personas líderes se centran más en aspectos relacionados con la enseñanza y el aprendizaje, principalmente en la gestión del aprendizaje y la mejora de las prácticas docentes.

Para Gajardo y Ulloa (2016), el liderazgo pedagógico, en términos generales, se enfatiza en un tipo de liderazgo escolar, cuyos propósitos son establecer objetivos educativos, planificar el currículo, evaluar a los docentes y la enseñanza, al igual que promover el desarrollo profesional docente.

En relación con los tipos de liderazgo, se muestran tres en los que el estudiantado realizó su práctica docente: 1. Liderazgo autocrático, donde el líder ejerce los niveles de poder sobre los subalternos. 2. Liderazgo no intervencionista (laissez-faire); es decir, la persona que está enfrente ejerce poca autoridad y deja que los demás sean los que lleven la iniciativa. 3. Liderazgo compartido, el cual, aunque toma las decisiones, tiene en cuenta lo que manifiestan las personas integrantes del equipo.

Además, Hernández (2013), sostiene que el liderazgo académico:

Es aquel que se manifiesta en la manera en que los investigadores definen la realidad en sus disciplinas o campos específicos de estudio y sostiene que los líderes académicos se caracterizan por ser mentes creativas capaces de abrir nuevas direcciones en la generación y transmisión del conocimiento (Hernández, 2013, p.1)

También es importante destacar los términos motivación y comunicación. Con respecto a este último, Müller (2013) considera que es transmitir mensajes utilizando un código o lenguaje previamente conocido por el emisor y el receptor y la motivación que se refiere al "Conjunto de factores internos o externos que determinan en parte las acciones de una persona" (Real Academia Española, 2014).

El liderazgo también tiene competencias, las cuales se refieren a las habilidades, destrezas, conocimientos y capacidades que posee determinada persona, que contribuyen al desarrollo de un liderazgo personal, entre ellas están las que mencionan Chinchilla, Jiménez, y García, (2018) las metacompetencias, entendiendo las que hacen referencia al desarrollo de la voluntad como (toma de decisiones, integridad, autocontrol y el equilibrio emocional). Lo anterior, se le atribuye a los comportamientos que coinciden con las virtudes de la prudencia, justicia, fortaleza entre otros (Chinchilla, Jiménez, y García, 2018, p.31)

Respecto al rendimiento académico, se define como la evaluación obtenida durante el proceso académico, para este estudio se considera además, a la capacidad de resolución, planificación didáctica, realización de tareas, proyectos, organización del tiempo, entre otros.

También como lo indica Ibáñez y Fuentes (2017):

Las Universidades deben convertirse en auténticos focos de liderazgo capaces de lograr los objetivos de desarrollo propuestos para este milenio, significa una nueva forma de comprender este nivel educativo y de descubrir en ella su enorme capacidad de desarrollo humano, ya que es la fuerza mayor para construir una sociedad del conocimiento inclusiva y diversa para avanzar en la innovación, investigación y creatividad, de ahí el innegable papel transformador que debe tener toda institución universitaria y en consecuencia la responsabilidad social que esta debe saber asumir (Ibáñez y Fuentes, 2017, p.186).

\section{Marco metodológico}

Este apartado muestra la metodología con base en la cual se desarrolló el estudio, según Abarca, et. al. (2013), consiste "en presentar los pasos, las herramientas de recolección de datos que serán utilizadas para alcanzar los objetivos propuestos" (Abarca, et. al. 2013, p. 59)

Al respecto, se consideraron el tipo de investigación, la población o muestra, fuentes de información e instrumentos para la recolección de los datos. Asimismo, se define cada uno de ellos.

El estudio se centra en un enfoque cualitativo. De acuerdo con Abarca et al. (2013), este refiere a "las relaciones entre las personas de la sociedad: lo que producen, dicen y lo que hacen frente y con los demás" (Abarca, et, al, 2013, p.11). Con base en la anterior definición, se considera la aplicación de este enfoque, debido a que las personas practicantes se relacionaron con estudiantes en el aula a la hora de impartir los contenidos, la realización de actividades y evaluaciones, entre otros. Se pretende así utilizar una metodología descriptiva e interpretativa con el propósito de conocer un liderazgo pedagógico desde la práctica docente.

Este tipo de indagación es fenomenológico porque describe y evidencia la realidad de aula, la experiencia de los estudiantes en su proceso de práctica docente, durante el I ciclo del 2017, la formación académica recibida desde el componente pedagógico y demás cursos durante la carrera.

La población seleccionada fue el grupo de estudiantes del curso, para un total de catorce personas y la muestra la conformaron trece. Dentro de las características de la población participante se destaca que la 
mayoría son personas becadas por la Universidad, por su condición económica y por ser de diferentes procedencias del país; sin embargo tres de ellas trabajan en el área secretarial.

El instrumento utilizado para la recolección de la información fue un cuestionario de preguntas abiertas y cerradas, con trece preguntas. En criterio de Abarca, et al. (2013), al formato de cuestionario se le conoce como "el conjunto de preguntas que se utilizan para interrogar a la gente sobre asuntos variados" (Abraca, et. al. 2013, p. 128).

Para medir cómo influye la calidad del liderazgo pedagógico de los docentes que trabajaron el curso en ese entonces, se tomó en cuenta el promedio de las calificaciones del estudiantado con el fin de evaluar el rendimiento académico. Además de analizar la percepción de la muestra con respecto a los docentes encargados del grupo y de los mismos participantes.

\section{Discusión y análisis de resultados}

De acuerdo con las respuestas obtenidas, la población estudiantil consultada definió el liderazgo como la acción de hacer las cosas de la mejor manera, motivando a los demás para hacerlo; la capacidad de convencer a otros de una manera positiva, característica de una persona de poder influir positivamente sobre un grupo de personas; buscar guiar a un grupo de personas o estar al mando de algo buscando el buen camino hacia el éxito de todas; característica que no todos los seres humanos poseen, esta funciona o se demuestra siendo un buen líder que saca lo mejor de su grupo, lo apoya a dar su máxima capacidad a base de buena motivación; persona que es un guía por seguir, que inspira por los alcances que ha tenido, sea profesional o personalmente; capacidad de dirigir, es la función que se le asigna a una persona; característica que marca las pautas para tomar un papel protagónico dentro de un equipo de trabajo; es un conjunto de habilidades para lograr diferentes objetivos o metas, ya sea de manera individual o en grupo.

De acuerdo con la descripción anterior sobre liderazgo, dada por los estudiantes esta tiene congruencia con la definición de Chinchilla, Jiménez y García (2018) ya que ambas indican que implica lucha, saber dirigir, es confiable y responde de manera ordenada ante cualquier situación.

Lo anterior refleja que el estudiante percibe que el docente debe tomar decisiones a la hora de trabajar en un ambiente de aula, también debe tener autocontrol para enfrentar diferentes situaciones. Además, ellos ven al personal académico como líder porque se muestra motivador, respetuoso, asertivo y capaz de trabajar con personas. También, si se compara el significado de la palabra educar con la percepción del estudiantado existe una gran relación porque para ellos ese término indica guiar y conducir, lo cual está muy ligado con el vocablo liderazgo, lo que significa que tanto una palabra como la otra están muy relacionadas.

Con respecto a los criterios plasmados por la muestra en cuanto a la motivación y la comunicación, ellos opinan que están muy relacionadas con el liderazgo porque se deben tener técnicas de comunicación muy asertivas para lograr la motivación en el grupo y guiarlo por el mejor camino.

Además la motivación es la causa para que la población practicante continúe con la carrera como se expresa la siguiente información:

- Las buenas experiencias que he tenido como docente, me siento enamorada de la profesión y me gustaría desempeñarla en el futuro.

- No dejar nada botado y terminar. Ganas de superación.

- Saber que puedo llegar a enseñarles a las personas más jóvenes, ser una herramienta para compartir conocimiento.

- Las estudiantes se han portado de buena forma, además de las docentes de la carrera en su mayoría han sido muy motivadoras y comprensivas.

- $\quad$ Me han motivado el poder expresarme con estudiantes, ayudándonos a ser mejores profesionales, y que no se incurra en los mismos errores que cometemos al iniciar nuestra etapa laboral.

- $\quad$ El deseo de trabajar en docencia.

- Siempre he querido ser docente.

- Me motivó a continuar con la carrera ya que la había dejado rezagada para concluir con otra carrera que llevaba.

- La actitud de los estudiantes y las ganas de ser una verdadera profesora.

- Las prácticas que he realizado han hecho darme cuenta de que es mi vocación.

De acuerdo con lo anterior, la motivación y la comunicación, según criterios del estudiantado, están muy relacionadas con el liderazgo porque la primera es la causa para que la población practicante continúe con la carrera ya que se deben tener técnicas de comunicación muy asertivas y buenos ejemplos de liderazgo pedagógico para lograr la motivación en cualquier grupo para encauzarlo y apropiarlo como futuros docentes.

Cuando se analiza lo que manifiesta Müller con respecto a la comunicación se cumple que entre los estudiantes practicantes y la docente hay comunicación ya que las estudiantes son emisores y la docente es 
el receptor, a su vez, entre ambos hay una realimentación porque se invierte el proceso de comunicación, en el caso del proceso de enseñanza y aprendizaje, la experiencia aprendida por los estudiantes los prepará para que en el futuro se desenvuelvan como docentes de Educación Comercial.

Con respecto a la motivación que perciben los estudiantes en el académico se encuentran valores, los intereses, aptitudes, la personalidad y habilidades intelectuales, lo cual se demuestra cuando ellos perciben factores internos que posee el docente cuando imparte el curso y que son claves para el desarrollo de la clase para despertar y continuar la motivación en el practicante.

Es importante destacar que la población consultada opina que el personal académico ha influido para llegar a la práctica docente:

- Al compartir el conocimiento sobre cómo desempeñarnos como docentes, motivación y dinamismo que hacen una clase más atractiva y nos dan ejemplos de cómo podemos ser como futuras académicas.

- Diplomado: son cursos fáciles. El bachillerato ha sido más difícil. La práctica con adultos y docente me ha ayudado para crecer profesionalmente.

- Muchísimo, me ayudan cada día, nunca me aburro con la materia que imparten ni con los métodos que utilizan.

- Siempre la mayoría de docentes me han aconsejado y brindado mucho apoyo con mi formación.

- Considero que poco, porque el personal académico es solo una guía; nosotros los estudiantes somos los que nos formamos mediante las prácticas y los profesores a los cuales ayudamos en la práctica.

- Mucho: hay profesores muy buenos que siempre nos motivan a seguir adelante.

- Me he topado con algunos profesores que me han motivado.

- Muy preocupadas para terminar y para que aprendiera.

- Con buenos consejos, brindando el apoyo académico y siendo guías de los distintos procesos.

- La totalidad ha influido para llegar a esta etapa de manera correcta.

De acuerdo con lo anterior, el estudiantado coincide en que el personal académico y la motivación tienen una gran influencia en el desarrollo de la práctica docente porque ambos inciden en la superación cuando se comparten conocimientos, se motiva y se reafirma la vocación del estudiante practicante. Además implica que cuanto más se potencie el liderazgo pedagógico con actividades, prácticas y estrategias mayor será la motivación, la comprensión y las herramientas que el futuro docente utilizará en sus clases, también se fomenta la confianza, las habilidades pedagógicas, la superación y la vocación de cada uno.

Relacionado con lo anterior está la evaluación final de cada estudiante, durante el proceso de su práctica docente, según se evidencia en la siguiente imagen.

\section{Calificación obtenida}

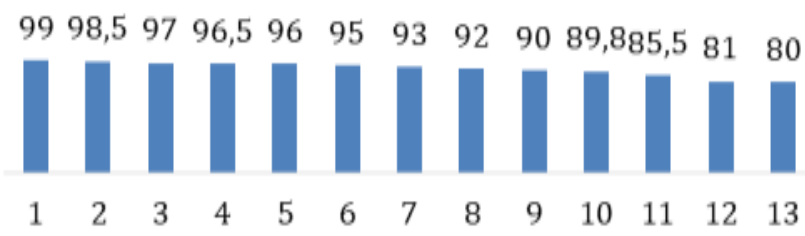

Figura 1. Notas obtenidas en el curso Fuente: Elaboración propia

Como se observa en la imagen anterior, la mayoría de los estudiantes obtuvieron notas de 80 en adelante, lo cual indica que pudieron terminar el curso y su práctica docente de manera satisfactoria porque lograron cumplir con los objetivos propuestos durante el curso, organización del tiempo, realización de planeamiento didáctico, horas de la práctica docente, resolución de conflictos en las diferentes instituciones educativas, cumplimiento de otros trabajos en el curso, entre otros.

También, entre los estudiantes practicantes se refleja el liderazgo pedagógico porque hay un vínculo entre ellos y el grupo en el que realizaron su práctica ya que se pone en práctica los conocimientos adquiridos durante la carrera, usan recursos didácticos y tiempo para preparar sus clases. Por eso se evidencia, que este proceso permitió a los practicantes la oportunidad de trabajar con sus capacidades pedagógicas y valorar si tenían vocación para desenvolverse en el campo de la docencia, pues hubo dos estudiantes que al finalizar el 
curso indicaron que se cambiarían de carrera. Actualmente, once del grupo de estudiantes están cursando la licenciatura de la misma carrera.

En cuanto a las características de un buen líder, la muestra mencionó las siguientes:

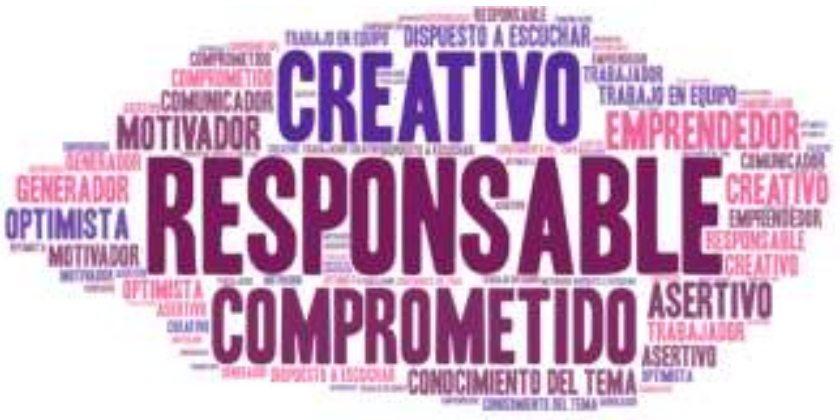

Figura 2. Características de un buen líder

Fuente: Elaboración propia, a partir de los resultados obtenidos

Como se observa en la figura anterior, las características de un buen líder que mayormente señalaron los encuestados fueron responsable, creativo, comprometido, motivador, optimista, emprendedor, asertivo, conocimiento del tema y dispuesto a escuchar.

Al tener el docente las características mencionadas, se refleja un liderazgo pedagógico en ese profesional porque tendrá hablidades, valores, destrezas y conocimiento para desarrollar actividades, estrategias que fortalezcan el proceso de enseñanza aprendizaje en el ambiente de aula, lo cual a su vez el practicante lo reflejará cuando sea un profesional en el área educativa.

Las opiniones de la población consultada con respecto al estilo de liderazgo en el personal académico se presentan en la tabla 1.

\begin{tabular}{|c|c|c|}
\hline $\begin{array}{c}\text { Estilo de } \\
\text { liderazgo }\end{array}$ & \multicolumn{1}{c|}{$\begin{array}{c}\text { Percepción del estudiantado sobre el estilo de liderazgo del personal } \\
\text { académico durante la carrera }\end{array}$} \\
\hline Compartido & $\begin{array}{c}\text { Debido a que los docentes siempre nos dan las indicaciones sobre lo que debemos } \\
\text { hacer, pero nos dan la libertad de trabajarlo de la forma que creamos la adecuada. }\end{array}$ \\
$\begin{array}{c}\text { Porque tienen el control y nos indican qué debemos hacer. } \\
\text { Nos indican qué hacer, pero también los estudiantes se proponen formas de } \\
\text { trabajar o se sienten seguros al decir que cosas no les parecen y podrían } \\
\text { cambiarse. }\end{array}$ \\
\hline $\begin{array}{l}\text { Estilo } \\
\text { autocrático }\end{array}$ & $\begin{array}{l}\text { Se enfoca más en que profesores le impongan al estudiantado las actividades que } \\
\text { deben realizar, sin tomar en cuenta sus decisiones o pensamientos. } \\
\text { Ya que siempre dependen de las decisiones de las autoridades mayores } \\
\text { (dirección) }\end{array}$ \\
\hline Laissez-faire & $-\begin{array}{l}\text { Nos dejan desenvolvernos con mucha libertad. } \\
\text { Ellos explican y después dejan que los estudiantes hagamos. }\end{array}$ \\
\hline
\end{tabular}

Tabla 1

Percepción de estilo de liderazgo en el personal académico

Fuente: Elaboración propia, a partir de los resultados obtenidos

En relación con la tabla 1, se evidencian los tres estilos del liderazgo en el proceso de formación de los estudiantes practicantes porque los docentes orientan, apoyan, escuchan, brindan la oportunidad de que ellos mismos puedan hacer, entonces es importante recordar que la forma de educación que se les brinde los hará independientes, críticos, motivación para utilizar tecnologías, tener valores, entre otros. Lo anterior refleja que el estudiantado resalta más el estilo de liderazgo compartido en el docente del curso de práctica docente, aunque también se da el autocritico en menor grado.

Los mismos estudiantes evalúan al personal académico como guías que comparten su conocimiento para desempeñarse como futuros docentes y desarrollan clases atractivas porque no se aburren, lo que los induce a crecer como futuros profesionales. Por eso, se manifiesta que esos docentes cumplen con el 
liderazgo pedagógico porque trabajan para transformar la sociedad cuando forman personas que serán los futuros formadores de la humanidad.

Por otra parte, la población practicante consultada declaró la relación del estilo de liderazgo con respecto al profesor colaborador, según la tabla 2.

\begin{tabular}{|c|c|}
\hline $\begin{array}{l}\text { Estilo de } \\
\text { liderazgo }\end{array}$ & $\begin{array}{c}\text { Percepción del estudiantado sobre el estidlo liderazgo del profesor } \\
\text { colaborador }\end{array}$ \\
\hline Laissez-faire & $\begin{array}{l}\text { - } \quad \text { Me permitió tener liderazgo en el aula y total libertad de buscar la información. } \\
\text { Siempre me apoyó en todo. } \\
\text { - } \quad \text { Daba la libertad de dar la clase, pero con ciertos lineamientos. } \\
\text { - } \quad \text { Ella dejó que yo hiciera todo y ella solo me acompañaba. } \\
\text { - } \quad \text { Siempre se me brindó la ayuda para tomar mis decisiones. } \\
\text { - } \quad \text { Me daba la libertad y decisión en las clases que yo iba a impartir. } \\
\text { - Él solo me brindó el tema y siempre me dejó hacer lo que yo creía correcto. } \\
\text { - Me daba la libertad y decisión en las clases que yo iba a impartir, además de los } \\
\text { permisos de los estudiantes mientras yo estuviera realizando mi práctica. }\end{array}$ \\
\hline Compartido & - $\quad$ Compartimos la manera de pensar y cómo hacer algunas cosas. \\
\hline $\begin{array}{l}\text { Autocrático y } \\
\text { compartido }\end{array}$ & - Había momentos en los cuales compartían el liderazgo. \\
\hline $\begin{array}{l}\text { Compartido, } \\
\text { laissez-faire }\end{array}$ & $\begin{array}{l}\text { - La profesora me dio el poder de la clase y me dio la libertad de realizar las } \\
\text { prácticas, actividades y materia a mi gusto. }\end{array}$ \\
\hline
\end{tabular}

Percepción del estilo de liderazgo en el profesor colaborador Fuente: Elaboración propia, a partir de los resultados obtenidos

La información de la tabla 2 evidencia que tiene relación con información de la tabla 1 porque los tres estilos de liderazgo son importantes para el proceso de enseñanza y aprendizaje y los tres tipos se vieron identificados durante la práctica docente. Como personas en el proceso de formación de los futuros docentes en el área de la Educación Comercial, se hace necesario guiar para la vida ya que esos aprendizajes recibidos serán los que los orienten a ejercer un mejor trabajo en el aula. Un excelente comentario de una estudiante practicante fue el siguiente: "cuando llegué a realizar la práctica docente, la profesora colaboradora me presentó como una colega, como una profesora, no como una practicante, ella me dio el lugar en el aula". Debido a esa experiencia, la estudiante se sintió parte del proceso. Actualmente, la joven es una profesora exitosa, recibe a practicantes de la carrera de Educación Comercial y los apoya.

Cuando se confrontan las opiniones anteriores con los autores Ibáñez y Fuentes (2017), la nueva forma de comprender la educación, la participación social, ser facilitador de los nuevos aprendizajes, se deduce que el estudiante practicante se cuestionará el para qué enseñar cuando sea formador de los conocimientos adquiridos en la vida universitaria y los asuma con responsabilidad social a la hora de ejercer su profesión.

Además, el estudiantado consideró que por medio de la práctica docente ha adquirido el liderazgo, lo cual se expresa en la siguiente figura.

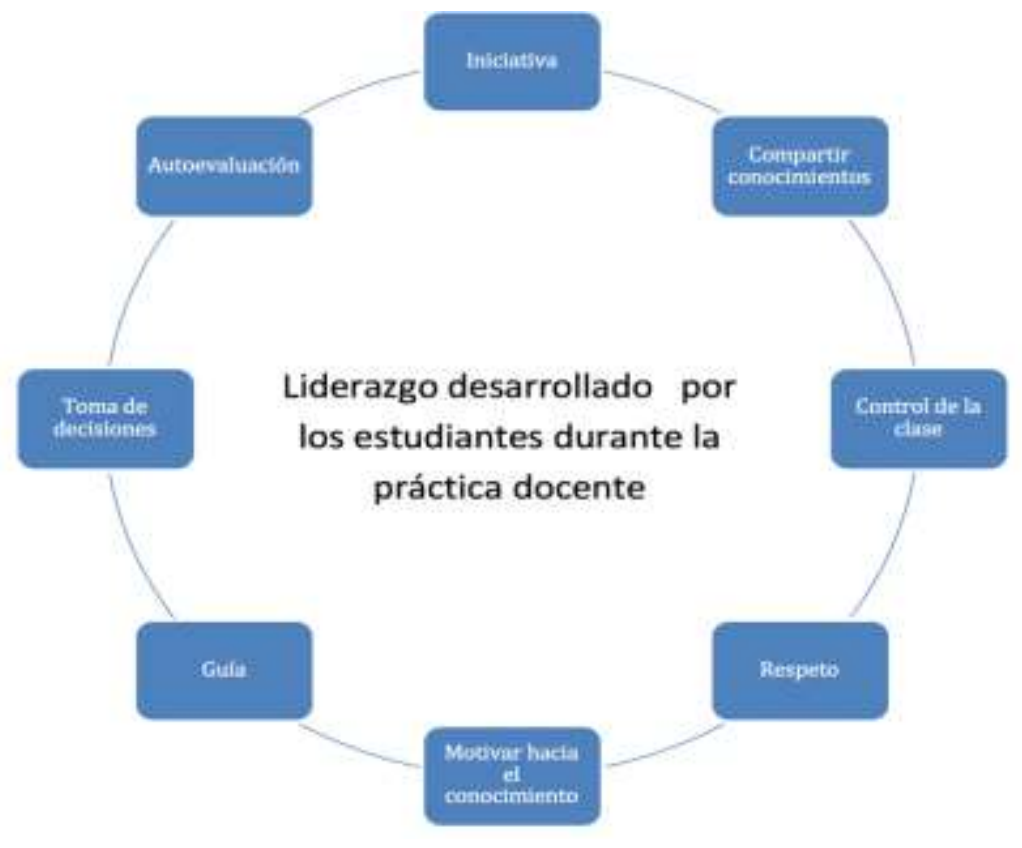


Figura 3. Liderazgo desarrollado durante la práctica docente Fuente: Elaboración propia, a partir de los resultados obtenidos

La población encuestada mencionó que adquirió liderazgo en el desarrollo de su práctica docente porque compartió conocimientos, tuvo iniciativa, logró controlar la clase, promovió valores, se motivó en aprender, fue guía, se autoevaluó en su proceso de enseñanza y aprendizaje, por ejemplo, al final del curso manifestaron lo siguiente: "profe me di cuenta que la docencia es lo que quiero, me di cuenta que la docencia no es lo mío", por lo tanto, con base en la figura anterior, se evidencia que alcanzaron obtener características que conducen al liderazgo y al liderazgo pedagógico por su desempeño de la práctica.

Las opiniones plasmadas por el estudiantado reflejan que como académicos en los cursos que se imparten en la carrera, al analizar los contenidos, la motivación, los valores que se promueven, se conduce a los estudiantes a promover la responsabilidad en la sociedad actual costarricense.

El hecho de que cada estudiante aplique actividades, prácticas, estrategias para desarrollar su clase durante la práctica docente implica que con su grupo de profesores formadores ha adquirido esas habilidades y destrezas del liderazgo pedagógico. Además, se infiere que el estudiantado adquiere autonomía para tomar decisiones y ponerlas en práctica como futuro docente en el área de Educación Comercial.

\section{Conclusiones}

De acuerdo con lo que se planteó en esta investigación, acerca de la influencia del liderazgo pedagógico en la práctica docente dirigida de la carrera de Educación Comercial de la Universidad Nacional, se concluye que la forma en que los docentes diseñen y organicen sus clases es la mejor influencia para que haya mayor aprendizaje de los estudiantes ya que el estudiantado tiene un gran potencial para transformar la sociedad cuando ejerza su profesión. Además, cuando se enfrenta al ambiente de aula, debe aceptar a los estudiantes como son, desarrollar relaciones humanas como la cortesía que son fundamentales en el proceso de enseñanza y aprendizaje y son clave para el resto de su vida profesional.

Además, se concluye que el estudiantado consideró que para que un líder desempeñe bien su trabajo debe tener capacitación, educación formal y reflexión de su práctica porque lo ayudará a resolver conflictos de la realidad cotidiana, aprender a desarrollarse en cada área, ser buenos líderes, tener vocación, conocimientos en tecnologías y en su especialidad y cualidades para desempeñar un buen trabajo y guiar a un grupo con éxito.

En el caso del curso Desafíos Didácticos, se refleja en las apreciaciones de los estudiantes practicantes porque se identificaron las características de un buen líder en el personal académico que orientó el proceso, tanto del docente del curso como del profesor colaborador que contribuyeron de una u otra forma para que la práctica docente se haya realizado satisfactoriamente.

Por otra parte, el estudiantado de práctica coincide en que el liderazgo es fundamental para la transformación, el enseñar debe realizarse con coherencia de acuerdo con las necesidades educativas que se viven a nivel de secundaria, es decir, llevar a cabo procesos innovadores y aprendizajes significativos para los nuevos ciudadanos. Asimismo, opina que no solo el liderazgo de los académicos, sino también la motivación y la comunicación asertiva son fundamentales para el proceso de enseñanza-aprendizaje.

El estudiantado al que se le aplicó el cuestionario considera que la comunicación y la motivación que han recibido en su formación académica son fundamentales para el desempeño de la práctica docente porque se relacionan con el liderazgo, por ejemplo, las características de un buen líder, son necesarias en el proceso de enseñanza-aprendizaje.

Las características y estilos de liderazgo van acompañados, como se pudo observar en los resultados, las primeras son necesarias en los nuevos docentes de educación comercial, para hacerle frente a las necesidades educativas que presentan los estudiantes y que responden para hacerlos más humanos y sensibles a la realidad social, en la cual se debe desarrollar un ambiente de permanencia de valores.

Con respecto a los estilos de liderazgo del personal académico, se reflejan tres: compartido, autocrático y laissez-faire; sin embargo, predominó el compartido.

En relación con los estilos de liderazgo que muestran los profesores colaboradores de las diferentes instituciones educativas, siete estudiantes manifestaron que se evidenció el estilo laissez-faire.

La población estudiantil que fue parte de la muestra refirió que se puso en evidencia un liderazgo en la práctica docente mediante la autoevaluación, la motivación del estudiantado a cargo y el desarrollo de actividades para facilitar el aprendizaje.

En cuanto a los aspectos relativos para que un líder desempeñe bien su trabajo, la población practicante considera lo siguiente: hacer bien su trabajo, estar capacitado en diferentes contenidos, reflexionar sobre su quehacer en la institución académica. 
REVISTA DE LA ESCUELA DE CIENCIAS DE LA EDUCACIÓN, AÑO 15, NRO. 14, VOL. 2, JULIO A DICIEMRE DE 2020. PÁGINAS 1-9. ISSN 2362-3349 (EN LÍNEA). INFLUENCIA DEL LIDERAZGO PEDAGÓGICO EN LA PRÁCTICA DOCENTE. MARÍA EUGENIA UGALDE VILLALOBOS. ALBA LUZ CANALES GARCÍA.

\section{Recomendaciones}

Las autoras presentan una parte inicial de la investigación, dado que permite profundizar en el futuro el tema con más población de estudiantes y docentes colaboradores del área de la Educación Comercial. Este trabajo realizado invita a continuar promoviendo liderazgo en la formación de formadores debido a que las competencias docentes actuales lo reflejan.

También es importante que la profesión docente investigue sobre los escenarios actuales, el ambiente, el autoconocimiento, los estilos de aprendizaje, la innovación, el manejo de emociones, apertura a la mejora continua y a tener la capacidad de aprender y desaprender, entre otras, ya que lo conducirá a ejercer un liderazgo oportuno y necesario en formación docente.

La comunicación y la motivación son fundamentales para obtener y desarrollar liderazgo en la formación docente de los futuros educadores de la Educación Comercial dado que las expectativas que tienen los estudiantes al ingresar a la carrera son muy altas, por ello la importancia de motivarles en contextos de formación reales para que cuando lleguen a sus prácticas puedan ejercer el liderazgo pedagógico.

\section{Referencias bibliográficas}

Abarca, A., et. al. (2013). Técnicas cualitativas de investigación. San José: EUCR.

Chinchilla, N.; Jiménez, E. y García, P. (2018). Integrar la vida. Barcelona: Ariel.

Gajardo, J. y Ulloa, J. (2016). El concepto de liderazgo pedagógico: tensiones y prácticas. En: Líderes Educativos. Centro Educativo para el Liderazgo Escolar. Recuperado de https://www.lidereseducativos.cl/wpcontent/uploads/2017/01/NT-6.pdf

Hernández, M. (2013). Liderazgo académico. En: Revista de la Educación Superior. Vol. XLII (3), N. 167, pp. 105131. Recuperado de http://publicaciones. anuies.mx/pdfs/revista/Revista167_S2A2ES.pdf

Ibáñez, J. y Fuentes, J. (2017). Educación y Capacidades: Hacia un nuevo enfoque del desarrollo humano. Madrid: Universidad Católica de Valencia.

Jové, R. (2017). La Escuela más feliz. Madrid: La Esfera de los libros.

Lecaros, R. (2017). Liderazgo pedagógico y desempeño docente en la Institución Educativa. Glorioso Húsares de Junín - El Agustino. [Tesis de maestría], Universidad César Vallejo. Recuperada de https://core.ac.uk/download/pdf/154579867.pdf

Müller, M. (2013). Curso básico de redacción. Costa Rica: EUCR.

Real Academia Española. (2014). Motivación. En: Diccionario de la Lengua Española (22ª ed.). Recuperado de https://dle.rae.es/?id=Pw7w4l0

Rodríguez, G. (2011). Funciones y rasgos del liderazgo pedagógico en los centros de enseñanza. En: Educación y Educadores, (14), n. 2, mayo-agosto, pp. 253-267. Recuperado http://educacionyeducadores.unisabana.edu.co/index.php/eye/article/view/1921/2510.

Villalobos, L. (2018). Enfoques y diseños de investigación Social. San José, Costa Rica: EUNED. 